\title{
The Cervical Range of Motion as a Factor Affecting Outcome in Patients With Congenital Muscular Torticollis
}

\author{
Jin-Youn Lee, MD, Seong-Eun Koh, MD, In-Sik Lee, MD, Heeyoune Jung, MD, \\ Jongmin Lee, MD, Jung-Il Kang, MD, Hyun Bang, MD
}

Department of Rehabilitation Medicine, Konkuk University School of Medicine and Konkuk University Medical Center, Seoul, Korea

Objective To investigate the factors affecting rehabilitation outcomes in children with congenital muscular torticollis (CMT).

Methods We retrospectively reviewed the medical records of 347 patients who were clinically suspected as having CMT and performed neck ultrasonography to measure sternocleidomastoid (SCM) muscle thickness. Fifty-four patients met the inclusion criteria. Included were demographic characteristics as well as measurements of cervical range of motion (ROM), SCM muscle thickness, and the abnormal/normal (A/N) ratio, defined as the ratio of SCM muscle thickness on the affected to the unaffected side.

Results Subjects were divided into three groups depending on degree of cervical ROM (group 1A: ROM>60, n=12; group 1B: $60 \geq R O M>30, n=31$; group 1C: $R O M \leq 30, n=11$ ), the $S C M$ muscle thickness (Th) (group 2A: Th $<1.2 \mathrm{~cm}$, $\mathrm{n}=23$; group 2B: $1.2 \leq \mathrm{Th}<1.4 \mathrm{~cm}, \mathrm{n}=18$; group $2 \mathrm{C}$ : $\mathrm{Th} \geq 1.4 \mathrm{~cm}, \mathrm{n}=13$ ), and the $\mathrm{A} / \mathrm{N}$ ratio (R) (group 3A: $\mathrm{R}<2.2, \mathrm{n}=19$; group 3B: $2.2 \leq R<2.8, n=20$; group $3 C$ : $R \geq 2.8, n=15$ ). We found that more limited cervical $R O M$ corresponded to longer treatment duration. The average treatment duration was 4.55 months in group 1A, 5.87 months in group $1 \mathrm{~B}$, and 6.50 months in group 1C. SCM muscle thickness and the $\mathrm{A} / \mathrm{N}$ ratio were not correlated with treatment duration.

Conclusion Infants with CMT who were diagnosed earlier and had an earlier intervention had a shorter duration of rehabilitation. Initial cervical ROM is an important prognostic factor for predicting the rehabilitation outcome of patients with CMT.

Keywords Congenital muscular torticollis, Range of motion, Rehabilitation, Prognosis

Received May 23, 2012; Accepted September 13, 2012 Corresponding author: Seong-Eun Koh

Department of Rehabilitation Medicine, Konkuk University Medical Center, 120 Neungdong-ro, Gwangjin-gu, Seoul 143-729, Korea Tel: +82-2-1588-1533, Fax: +82-2-2030-5379, E-mail: kohse@kuh.ac.kr

(c) This is an open-access article distributed under the terms of the Creative Commons Attribution Non-Commercial License (http://creativecommons. org/licenses/by-nc/3.0) which permits unrestricted noncommercial use, distribution, and reproduction in any medium, provided the original work is properly cited.

Copyright $\odot 2013$ by Korean Academy of Rehabilitation Medicine

\section{INTRODUCTION}

Torticollis is one of the most common types of abnormal head and neck postures in infants. Torticollis was first defined by Tubby in 1912 as "A deformity, either congenital or acquired, characterized by lateral inclination of the head to the shoulder, with torsion of the neck and deviation of the face" [1]. The term congenital muscular torticollis (CMT) suggests a neck deformity primarily in- 
volving the shortening of the sternocleidomastoid (SCM) muscle that is detected at birth or shortly after birth [1]. CMT is the most common form of torticollis in children, significantly outnumbering orthopedic, neurologic, and ocular causes $[1,2]$.

MacDonald classified patients with CMT into two groups. First are those patients with a palpable sternomastoid tumor $[2,3]$. A pseudotumor or SCM tumor, also known as fibromatosis colli, typically presents as a painless, firm, fibrous mass in the SCM muscle in infants $2-4$ weeks of age [4]. The second group consists of patients with tightness of the SCM muscle but no palpable tumor, who are designated as having idiopathic muscular torticollis. Postural torticollis, another type of torticollis, is a term used to describe patients with congenital torticollis who have all of the clinical features of torticollis but no demonstrable tightness or tumor of the muscle [5]. In the current study, the first two groups, including patients with a palpable sternomastoid tumor or tightness of the SCM muscle, are regarded as categories of CMT.

The incidence of torticollis varies widely, but can be as high as 19 per 1,000 live births [2]. According to Porter and Blount [4], pseudotumors occur in approximately $0.4 \%$ of all newborns; the mass usually increases in size until 1 month of age, diminishes gradually, and disappears after 3 months of age. However, $10 \%-20 \%$ of infants with an SCM tumor develop CMT as a result of fibrosis of the remaining tumor. Although SCM tumors and CMT are regarded as different disease entities in Stuart's study, the etiology of these two conditions are explained as part of the same disease spectrum [4]. Histologically, CMT is recognized as endomysial fibrosis with the deposition of collagen and the migration of fibroblasts around individual muscle fibers [6]. This finding is in accordance with the derivation of CMT from the fibrosis of an SCM tumor.

Although most infants with CMT show a rapid resolution of the SCM mass during early infancy, a few fail to recover full cervical range of motion (ROM) during their childhood [7]. Many studies that evaluated the natural history and outcomes of children with CMT produced varied results, depending on the type, severity, and age at diagnosis of torticollis [1]. Generally, with conservative treatment, patients with postural torticollis do better than those with muscular torticollis, and patients with only SCM muscle tightness do better than those with obvious fibrotic tumor of the SCM muscle [2]. Conservative treatment, consisting of manual stretching and massage, has proven effective. Cheng et al. [7] did a prospective study of 510 infants with SCM pseudotumor and CMT and found that $90.7 \%$ of subjects had excellent and good overall results after a manual stretching program.

It is believed that the success of conservative treatment depends on the patient's age at the time of presentation. Earlier ultrasonography studies demonstrated that patients with severe fibrosis were likely to be older than those with mild fibrosis [6].

However, previous studies that tried to determine the influence of other clinical features of CMT-such as cervical ROM, SCM muscle thickness, and relative size of a mass to the unaffected side-to the outcome of conservative treatment produced inconsistent results. Therefore, the current study was designed to investigate the factors affecting rehabilitation outcomes in children with CMT.

\section{MATERIALS AND METHODS}

\section{Subjects}

We identified 347 infants with clinically suspected torticollis with abnormal neck posture who visited a rehabilitation clinic at a tertiary medical center from March 2008 to March 2012, and screened the subjects using neck ultrasonography. Patients younger than 3 months of age with a palpable neck mass or tightness and limited passive cervical ROM were included in this study. Patients with congenital anomalies of the cervical spine or ocular anomalies were excluded. Subjects with benign paroxysmal torticollis were also excluded. Among the 347 infants, 54 (30 males and 24 females) met the inclusion criteria. Of the remaining 293 patients, 203 had another type of torticollis, such as benign paroxysmal torticollis or ocular abnormality, and 90 patients were excluded because they had incomplete medical records or had not completed the rehabilitation program. The study was reviewed and approved by Institutional Review Board, and informed consent was obtained from all the parents or caregivers of the patients.

\section{Methods}

We obtained plain radiographs of the cervical spine with anterior-posterior and lateral views to exclude any spinal abnormalities. Neck ultrasonography was performed by 2 physicians with 2 years experience to con- 

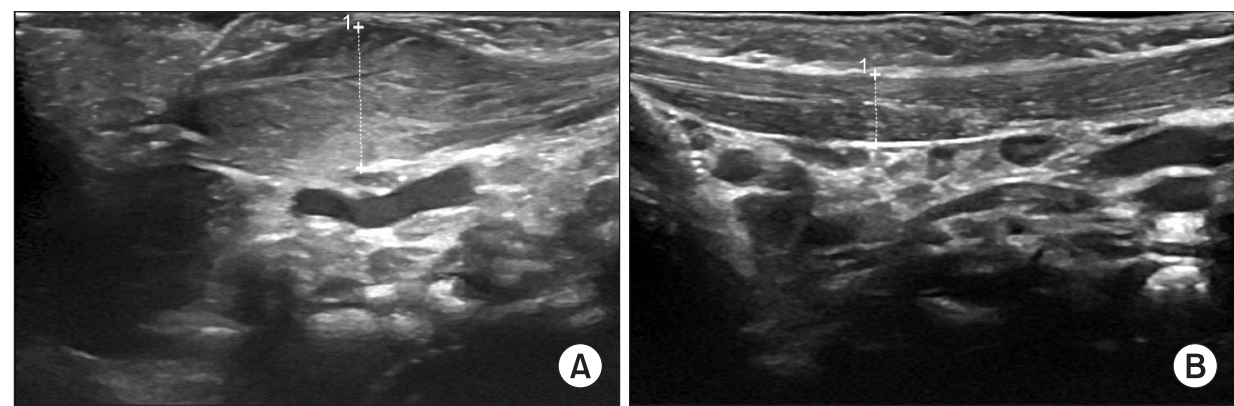

Fig. 1. Ultrasonographic features of sternocleidomastoid muscle with longitudinal view in a child with congenital muscular torticollis. (A) Sternocleidomastoid muscle of affected side shows heterogenic echotexture and fusiform dilatation. (B) Sternocleidomastoid muscle of unaffected side shows homogenous echotexture.

Table 1. Demographic description of participants

\begin{tabular}{lc}
\hline \multicolumn{1}{c}{ Variable } & Value \\
\hline Sex & \\
Male & $30(55.6)$ \\
\hline Female & $24(44.4)$ \\
Mass side & $32(59.3)$ \\
\hline Right & $22(40.7)$ \\
\hline Left & \\
Mode of delivery & $44(81.5)$ \\
\hline NSVD & $10(18.5)$ \\
\hline Cesarean section & $39.26 \pm 1.05$ \\
Gestational age (wk) & $3.28 \pm 0.38$ \\
\hline Birth weight (kg) & $36.63 \pm 14.80$ \\
\hline Age at diagnosis (day) & $49.35 \pm 17.16$ \\
Cervical ROM $\left({ }^{\circ}\right)$ & $1.24 \pm 0.24$ \\
\hline SCM muscle thickness $(\mathrm{cm})$ & $2.48 \pm 0.66$ \\
\hline Abnormal/normal ratio & \\
\hline
\end{tabular}

Values are presented as mean \pm standard deviation or number (\%).

NSVD, normal spontaneous vaginal delivery; ROM, range of motion; SCM, sternocleidomastoid.

firm the existence of the neck mass or hypertrophy of the SCM muscle (Fig. 1). We measured bilateral SCM muscle thickness on the longitudinal view at the largest dimension by scanning along the SCM muscle between the level of the mastoid process and the level of the junction between the sternum and the clavicle; scans were performed three times for each subject, and averaged. The patients were examined in the supine position with their necks slightly extended by putting a small pillow underneath the shoulder and rotating the head to the opposite side.

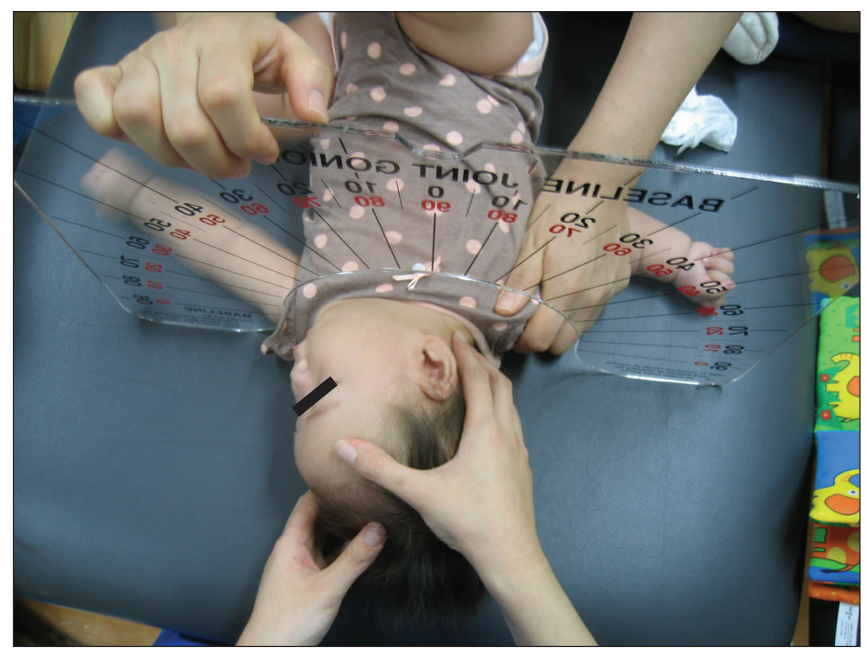

Fig. 2. Measurement of range of motion of cervical rotation.

Sex, gestational age, birth weight, mode of delivery, and affected side were determined from the patients' medical records. In addition, we measured SCM muscle thickness, cervical ROM, and the abnormal/normal (A/ $\mathrm{N})$ ratio, defined as the ratio of the SCM thickness on the affected to the unaffected side. These characteristic data are shown in Table 1.

Conservative physiotherapy including manual stretching exercises, massage, strengthening exercises of the unaffected side, and therapeutic ultrasonography was performed on each patient and a standardized physiotherapy program-3 times a week for 30 minutes per session-was performed by an experienced physical therapist. Measurement of cervical ROM was done once a month and diagnostic ultrasonography was performed at 2- to 3-month intervals, according to the improvement of clinical features (Fig. 2). 
Physical treatment was initiated soon after the subjects were diagnosed with CMT. The end points of the study were defined as the time when the cervical ROM was normalized or the deficits in the cervical $\mathrm{ROM}$ were $5^{\circ}$ or less, when no palpable mass remained, and when the difference in the SCM muscle thickness of the affected and unaffected sides was less than $2 \mathrm{~mm}$ [8]. Deficits in cervical ROM that were greater than $5^{\circ}$ at the age of 1 year, or no further improvement after successive treatment up to the age of 1 year, were regarded as treatment failure $[8,9]$. Treatment duration was defined as the time between the first and the last day of standardized physiotherapy, at which time the initial and final assessments were done.

For this study, patients were divided into three groups depending on the initial degree of passive cervical rotation ROM (group 1A, ROM $>60$; group $1 \mathrm{~B}, 60 \geq \mathrm{ROM}>30$; group $1 \mathrm{C}, \mathrm{ROM} \leq 30$ ), SCM muscle thickness (Th) (group $2 \mathrm{~A}, \mathrm{Th}<1.2 \mathrm{~cm}$; group $2 \mathrm{~B}, 1.2 \leq \mathrm{Th}<1.4 \mathrm{~cm}$; group $2 \mathrm{C}$, $T h \geq 1.4 \mathrm{~cm}$ ), and the $A / N$ ratio $(R)$ (group $3 A, R<2.2$; group $3 \mathrm{~B}, 2.2 \leq \mathrm{R}<2.8$; group $3 \mathrm{C}, \mathrm{R} \geq 2.8$ ).

\section{Statistical analysis}

The Spearman rank correlation coefficient was used to assess the association between the variables observed and treatment durations. The Mann-Whitney U-test was used to compare means for each group according to sex, side of the mass, and mode of delivery. SPSS ver. 17.0 (SPSS Inc., Chicago, IL, USA) statistical software was used in the analysis. Null hypotheses of no difference were rejected if $\mathrm{p}$-values were less than 0.05 .

Table 2. Duration of conservative physiotherapy by demographic factors

\begin{tabular}{lcc}
\hline \multicolumn{1}{c}{ Variable } & Value & p-value \\
\hline Sex & & 0.705 \\
\hline Male & $5.53 \pm 2.37$ & \\
$\quad$ Female & $5.79 \pm 2.40$ & \\
Mass side & & 0.062 \\
$\quad$ Right & $6.19 \pm 2.53$ & \\
Left & $4.86 \pm 1.88$ & \\
Mode of delivery & & 0.848 \\
$\quad$ NSVD & $5.66 \pm 2.30$ & \\
\hline Cesarean section & $5.60 \pm 2.76$ & \\
\hline
\end{tabular}

NSVD, normal spontaneous vaginal delivery. $\mathrm{p}<0.05$ was considered significant. Statistical significance was evaluated using the Mann-Whitney U-test.

\section{RESULTS}

Fifty-four infants (30 males and 24 females) whose ages ranged from 13 to 70 days (mean \pm standard deviation [SD], 36.62 \pm 14.80 days) were included in this study. The basic clinical characteristics are shown in Table 1 . The right side was affected more often than the left side ( $59.26 \%$ vs. $40.74 \%$, respectively). The majority of the subjects $(81.48 \%)$ were born via normal spontaneous vaginal delivery and the remaining $18.52 \%$ were by cesarean section. Mean gestational age was 39.26 weeks (SD, \pm 1.05 ) and the mean birth weight was $3.28 \mathrm{~kg}$ (SD, \pm 0.38 ). Age at diagnosis averaged 36.62 days $(\mathrm{SD}, \pm 14.80)$ and the mean cervical ROM at the time of diagnosis was $49.35^{\circ}$ (SD, \pm 17.16 ). The average thickness of the SCM muscle was $1.24 \mathrm{~cm}(\mathrm{SD}, \pm 0.24)$ and the $\mathrm{A} / \mathrm{N}$ ratio was 2.48 (SD, \pm 0.66 ). Among these characteristics, sex, the side of the mass, and the mode of delivery were not significantly correlated with treatment duration (Table 2). Gestational age and birth weight also were not significantly correlated with treatment duration (Table 3).

The rehabilitation program using conservative physical therapy was completed in $52(96.30 \%)$ of the 54 total cases and 2 patients (3.70\%) did not reach full cervical ROM before 1 year of age. The mean age at the time of diagnosis of the two patients who failed to recover full cervical ROM before the age of 1 year was 46 days (51 days and 41 days); this was relatively older than the mean age of all of the subjects. Both of these patients presented with a right-sided SCM muscle mass and showed severe limitations in cervical $\mathrm{ROM}\left(20^{\circ}\right.$ and $10^{\circ}$, respectively), which were classified as group 1C. Their SCM muscle thicknesses were greater than average $(1.8 \mathrm{~cm}$ and $1.54 \mathrm{~cm}$, respectively) and were classified as group $2 \mathrm{C}$, although it was not statistically significant. In addition, one of the patients showed a high $\mathrm{A} / \mathrm{N}$ ratio, 2.95, which was classified as $3 \mathrm{C}$, but the ratio of the other, 2.18 , was classified

Table 3. Demographic factors and durations of conservative physiotherapy

\begin{tabular}{lcc}
\hline \multicolumn{1}{c}{ Variable } & $\begin{array}{c}\text { Correlation } \\
\text { coefficient }\end{array}$ & p-value \\
\hline Gestational age (wk) & 0.091 & 0.309 \\
Birth weight $(\mathrm{kg})$ & -0.136 & 0.164 \\
Age at diagnosis (day) & 0.259 & $0.029^{*}$ \\
\hline $\mathrm{p}<0.05,{ }^{*} \mathrm{p}<0.01$, Spearman correlation. &
\end{tabular}


as $3 \mathrm{~A}$, although this was also not significant.

The age at the time of diagnosis was positively correlated with total treatment duration (correlation coefficient, 0.259; $\mathrm{p}=0.029$ ) (Table 3). The total treatment duration was compared among the groups, and the groups were classified according to the age at diagnosis (group A: age $\leq 21$ days, $n=8$; group $B$ : $21 \leq$ age $<42$ days, $n=32$; group $C$, age $>42$ days, $n=14$ ). We found significant correlations between the age at diagnosis and the total treatment duration, shown in Table 4.

We investigated three variables-cervical ROM, SCM muscle thickness, and the $\mathrm{A} / \mathrm{N}$ ratio at the time of diagnosis-that are considered to influence treatment duration (Table 5). Cervical ROM was divided into three groups: group $1 \mathrm{~A}, \mathrm{ROM}>60^{\circ}, \mathrm{n}=12$; group $1 \mathrm{~B}, 30^{\circ} \leq \mathrm{ROM}<60^{\circ}$, $\mathrm{n}=31$; and group $1 \mathrm{C}, \mathrm{ROM} \leq 30^{\circ}, \mathrm{n}=11$. The average duration of treatment was 4.09 in group 1A, 5.87 in group 1B, and 6.50 months in $1 \mathrm{C}$, with statistically significant correlations (correlation coefficient, $0.307 ; \mathrm{p}=0.012$ ). Thickness of the SCM muscle, which was measured by ultrasonography using a long-axis view, was classified into three groups: group $2 \mathrm{~A}, \mathrm{Th}<1.2 \mathrm{~cm}, \mathrm{n}=23$; group $2 \mathrm{~B}, 1.2 \leq \mathrm{Th}<1.4$ $\mathrm{cm}, \mathrm{n}=18$; and group $2 \mathrm{C}, \mathrm{Th} \geq 1.4 \mathrm{~cm}, \mathrm{n}=13$ ). The average duration of treatment for groups $2 \mathrm{~A}$ was $5.65,2 \mathrm{~B}$ was 5.78 , and $2 \mathrm{C}$ was 5.46 months, with no significant differences between groups (correlation coefficient, $-0.025 ; \mathrm{p}=0.429$ ). The $\mathrm{A} / \mathrm{N}$ ratio was subdivided into three groups (group $3 A$ : $R<2.2, n=19$; group $3 B: 2.2 \leq R<2.8, n=20$; and group $3 C: R \geq 28, n=15)$. The average treatment duration for group $3 \mathrm{~A}$ was 6.11 , for $3 \mathrm{~B}$ was 5.30 , and for $3 \mathrm{C}$ was 5.53 months, and there were no significant differences between groups (correlation coefficient, $-0.088 ; p=0.262$ ). In addition, there were no significant correlations between the cervical ROM and the $\mathrm{A} / \mathrm{N}$ ratios (correlation coefficient, $0.073 ; \mathrm{p}=0.300)$.

Treatment duration (Dur) was classified into three groups (group A: Dur $\leq 4, n=16$; group B: $4<$ Dur $\leq 8, n=32$; and group C: Dur $>8, n=6$ ) and the initial cervical ROM, SCM muscle thickness, and A/N ratio were compared among groups. We found significant correlations between treatment duration and cervical ROM, with a longer treatment duration corresponding to a more se-

Table 4. Duration of conservative physiotherapy by age at diagnosis

\begin{tabular}{ccccc}
\hline Group & Age at diagnosis (day) & Treatment duration (mo) & Correlation coefficient & p-value \\
\hline A & $\leq 21$ & $3.88 \pm 1.73$ & - & - \\
B & $21<\& \leq 42$ & $5.84 \pm 2.37$ & 0.292 & $0.016^{*}$ \\
C & $42>$ & $6.21 \pm 2.33$ & - & - \\
\hline
\end{tabular}

Values are presented as mean \pm standard deviation.

$\mathrm{p}<0.05,{ }^{*} \mathrm{p}<0.01$, Spearman correlation.

Table 5. Duration of conservative physiotherapy by group

\begin{tabular}{|c|c|c|c|}
\hline Group & Treatment duration (mo) & Correlation coefficient & p-value \\
\hline Group 1: cervical ROM $\left(^{\circ}\right)$ & & 0.307 & $0.012^{*}$ \\
\hline $1 \mathrm{~A}(>60)$ & $4.09 \pm 1.58$ & & \\
\hline $1 \mathrm{~B}(60 \geq \&>30)$ & $5.87 \pm 2.14$ & & \\
\hline $1 C(\leq 30)$ & $6.50 \pm 2.97$ & & \\
\hline Group 2: SCM muscle thickness (cm) & & -0.025 & 0.429 \\
\hline $2 \mathrm{~A}(<1.2)$ & $5.65 \pm 2.52$ & & \\
\hline $2 \mathrm{~B}(1.2 \leq \&<1.4)$ & $5.78 \pm 2.05$ & & \\
\hline $2 \mathrm{C}(\geq 1.4)$ & $5.46 \pm 2.67$ & & \\
\hline Group 3: abnormal/normal ratio & & -0.088 & 0.262 \\
\hline $3 \mathrm{~A}(<2.2)$ & $6.11 \pm 2.56$ & & \\
\hline 3B $(2.2 \leq \&<2.8)$ & $5.30 \pm 2.20$ & & \\
\hline $3 C(\geq 2.8)$ & $5.53 \pm 2.39$ & & \\
\hline
\end{tabular}

ROM, range of motion; SCM, sternocleidomastoid.

${ }^{*} \mathrm{p}<0.05$, Spearman correlation. 
Table 6. Initial cervical ROM, SCM thickness, and A/N ratio by treatment duration

\begin{tabular}{lccccc}
\hline & Group A & Group B & Group C & $\begin{array}{c}\text { Correlation } \\
\text { coefficient }\end{array}$ & p-value \\
\hline Treatment duration $(\mathrm{mo})$ & $\leq 4$ & $4<\& \leq 8$ & $>8$ & & \\
Cervical ROM $\left(^{\circ}\right)$ & $56.25 \pm 17.65$ & $49.53 \pm 14.94$ & $30.00 \pm 14.14$ & -0.387 & $0.002^{*}$ \\
SCM thickness $(\mathrm{cm})$ & $1.26 \pm 0.29$ & $1.23 \pm 0.20$ & $1.19 \pm 0.28$ & -0.058 & 0.339 \\
A/N ratio & $2.50 \pm 0.73$ & $2.47 \pm 0.65$ & $2.45 \pm 0.70$ & -0.015 & 0.459 \\
\hline
\end{tabular}

ROM, range of motion; SCM, sternocleidomastoid; A/N, abnormal/normal ratio.

${ }^{*} \mathrm{p}<0.05$, Spearman correlation.

vere limitation in cervical ROM (correlation coefficient, -0.387; $\mathrm{p}=0.002$ ). Two other variables, SCM thickness and $\mathrm{A} / \mathrm{N}$ ratio, did not have significant correlations by treatment duration (Table 6).

\section{DISCUSSION}

CMT is one of the most common causes of neck-posture abnormalities in children, and resolves spontaneously within a few months with good prognosis. Nevertheless, surgical intervention is necessary in some recalcitrant cases if the child cannot achieve full cervical ROM and deficits remain despite intensive physical therapy including manual stretching and massage. Therefore, it is important to find factors that may influence rehabilitation outcomes in patients with CMT, to avoid invasive procedures and to achieve better outcomes.

In this study, we evaluated the relationships among different demographic factors and the duration of a rehabilitation program. Demographic findings showed a slight male predominance (male, 55.6\%; female, $44.4 \%$ ) and that the right side is more commonly affected (59.26\%), as noted in previous studies [10].

Neck-motion abnormalities in children with CMT are presented as limitations in lateral bending as well as in cervical rotation. Although both lateral bending and cervical rotation measurements indicate the severity of a neck-motion abnormality, a previous study suggested that the measurement of cervical rotation has better inter-examiner reliability than a side-bending measurement [5]. Therefore, the degree of cervical rotation was adopted as a predictor of treatment outcome in this study. The results showed that a severe deficit in cervical ROM results in longer physical therapy. Cheng et al. [7] suggested that cervical ROM deficits influenced treatment duration. They subdivided subjects with deficits in cervical ROM into four groups: group I, $0^{\circ}$; group II, $1^{\circ}-$ $15^{\circ}$; group III, $16^{\circ}-30^{\circ}$; and group IV, $>30^{\circ}$. Groups III and IV showed the longest treatment duration (5.2 months) compared to the other groups. In Cheng's study [7], deficits in cervical ROM of $30^{\circ}$ or less were subdivided into three groups and deficits greater than $30^{\circ}$ were assessed as one group; the researchers did not suggest the differences in treatment durations according to severities of limitation in cervical ROM within the group. In the current study, subjects with more severe deficits in cervical ROM were included. We divided groups according to cervical $\mathrm{ROM}$, with groups $\mathrm{BB}$ and $\mathrm{IC}$ representing deficits in cervical ROM greater than $30^{\circ}$, and group $1 \mathrm{C}$ with deficits greater than $60^{\circ}$ showed the longest treatment.

The A/N ratio, defined as the ratio of SCM muscle thickness on the affected side to the unaffected side, was measured. SCM muscle thickness varies in size in each subject depending on developmental factors such as aging, sex, and other innate variables, so that it is sometimes misleading to compare the raw thickness. The A/ $\mathrm{N}$ ratio, which indicates the relative thickness of affected SCM muscle in CMT patients assuming that the SCM thickness of the unaffected side is normal, is a reasonable and convenient method to compare the relative degree of muscle hypertrophy. Han et al. [11] showed that the ratio of the thickness of the SCM muscle on the lesion side to the non-lesion side, as well as the raw SCM muscle thickness, was correlated with the duration of rehabilitation. Lim et al. [12] divided treatment duration into two groups, one less than 12 months and the other greater than 12 months, and analyzed the correlations with the $\mathrm{A} / \mathrm{N}$ ratio. The results showed that the groups with treatment duration longer than 12 months had a greater $\mathrm{A} / \mathrm{N}$ ratio in terms of SCM muscle thickness when measured longitudinally, although there was no statistically significant difference in the $\mathrm{A} / \mathrm{N}$ ratio determined by measur- 
ing the cross-sectional circumference [12]. In the current study, however, neither the A/N ratio nor SCM muscle thickness were related to treatment duration.

We found that infants who were diagnosed earlier with CMT had a shorter length of treatment. This result is in agreement with previous studies. Tatli et al. [8] subdivided subjects with sternomastoid tumor and postural torticollis into two groups according to the age at presentation-less than 6 weeks and between 6 and 24 weeksand found that patients who presented with a sternomastoid tumor or postural torticollis at less than 6 weeks old had a shorter period of therapy compared to those with the later presentation. Petronic et al. [13] subdivided patients diagnosed with CMT into five groups according to their age at first visit and showed that earlier treatment is shorter in duration and more cost-effective.

The present study had limitations that should be considered. First, the severity of fibrosis on ultrasonography was not considered a prognostic factor in this study. Kim et al. [14] divided ultrasonographic findings of CMT into five subgroups, according to echogenicity and the presence of a mass, and found that grades $3-5$, representing a more severe type of CMT, had longer treatment compared to grades 1 and 2. However, Lim et al. [12] found that the echogenicity of the affected SCM muscle was not correlated with the duration of treatment. Ultrasonographic findings that show the patterns of muscle fibrils and different echogenicity according to the different degrees of severity should be considered in future studies.

Second, the location of a mass in the SCM muscle was considered influential on the rehabilitation outcome. In the current study, SCM muscle thickness was measured from the site that shows the largest diameter with scanning along the SCM muscle from the level of the mastoid process to the level of the sternum and clavicle. Cheng et al. [7] adopted a scoring system for evaluating the outcomes of children with CMT. Among their evaluation categories was the presence of a residual band, scored according to its location in the SCM muscle: no band ( 3 points), lateral ( 2 points), lateral and cleidal (1 point), and cleidal and sternal (0 points). Higher scores represented good prognoses. However, in their study, determining the location of a residual band was done by palpating the SCM muscle and was not confirmed with ultrasonography. Measuring SCM muscle thickness at the level of the clavicle and sternum using a longitudinal view is difficult because bony structures obscure the full thickness of the muscle. Instead, an axial view to measure the SCM muscle circumference is a more accurate method for determining muscle hypertrophy. In future studies, ultrasonography should be used to determine the precise locations of masses or the largest dimension of the muscle thickness before and after treatment, and the relationship of these measurements to the rehabilitation outcome should be included. Furthermore, comparing SCM muscle thickness using a longitudinal view as well as an axial view will be helpful in determining the severity of CMT.

Third, a follow-up evaluation was not done after the completion of rehabilitation therapy, and it is not known whether the effects of treatment have been maintained. In addition, home exercise after each session of the rehabilitation program was not controlled, so it is possible that some subjects received more intensive manual stretching and massage at home. Inter-therapist variability in performing manual stretching and massage is another possible confounding factor that should be considered. Therefore, treatment protocols should be applied in more controlled circumstances in future studies.

Our study's small sample size lessened its statistical power. Congenital abnormalities and birth-related problems that are known to be related to CMT such as hip dysplasia, plagiocephaly, or clavicle fracture were not presented by our subjects. A prospective study with a large sample size that represents the general population would be helpful for analyzing prognostic factors of CMT and increasing statistical power.

Although our study contains some limitations, it is significant that we identified limitations in cervical ROM as a reliable prognostic factor for the outcomes of patients with CMT. The positive correlations between limitations in cervical ROM and treatment duration were confirmed even in infants with more severe limitations of greater than $30^{\circ}$. Even in patients with severe limitations in cervical rotation, the prognosis is good in CMT. Therefore, early diagnosis and the early initiation of physical treatment are necessary in infants who are clinically suspected of having CMT. In addition, despite the presentation of severely limited cervical rotation, conservative manual therapy should be tried first before considering invasive procedures on infants.

In conclusion, infants with CMT who were diagnosed 
earlier and had an earlier intervention had a shorter duration of physical therapy. This result agrees with previous studies. In addition, the initial ROM of cervical rotation is important for predicting the duration of physical therapy in patients with CMT.

\section{CONFLICT OF INTEREST}

No potential conflict of interest relevant to this article was reported.

\section{REFERENCES}

1. Cheng JC, Tang SP, Chen TM, Wong MW, Wong EM. The clinical presentation and outcome of treatment of congenital muscular torticollis in infants: a study of 1,086 cases. J Pediatr Surg 2000;35:1091-6.

2. Joyce MB, de Chalain TM. Treatment of recalcitrant idiopathic muscular torticollis in infants with botulinum toxin type A. J Craniofac Surg 2005;16:321-7.

3. Macdonald D. Sternomastoid tumour and muscular torticollis. J Bone Joint Surg Br 1969;51:432-43.

4. Porter SB, Blount BW. Pseudotumor of infancy and congenital muscular torticollis. Am Fam Physician 1995;52:1731-6.

5. Cheng JC, Wong MW, Tang SP, Chen TM, Shum SL, Wong EM. Clinical determinants of the outcome of manual stretching in the treatment of congenital muscular torticollis in infants: a prospective study of eight hundred and twenty-one cases. J Bone Joint Surg Am 2001;83-A:679-87.

6. Lee YT, Yoon K, Kim YB, Chung PW, Hwang JH, Park YS, et al. Clinical features and outcome of physiother- apy in early presenting congenital muscular torticollis with severe fibrosis on ultrasonography: a prospective study. J Pediatr Surg 2011;46:1526-31.

7. Cheng JC, Tang SP, Chen TM. Sternocleidomastoid pseudotumor and congenital muscular torticollis in infants: a prospective study of 510 cases. J Pediatr 1999;134:712-6.

8. Tatli B, Aydinli N, Caliskan M, Ozmen M, Bilir F, Acar G. Congenital muscular torticollis: evaluation and classification. Pediatr Neurol 2006;34:41-4.

9. Wei JL, Schwartz KM, Weaver AL, Orvidas LJ. Pseudotumor of infancy and congenital muscular torticollis: 170 cases. Laryngoscope 2001;111(4 Pt 1):688-95.

10. Do TT. Congenital muscular torticollis: current concepts and review of treatment. Curr Opin Pediatr 2006;18:26-9.

11. Han SJ, Shin BM, Lee JM, Yoon TS. Factors affecting rehabilitation outcome of congenital muscular torticollis. J Korean Acad Rehabil Med 2010;34:643-9.

12. Lim D, Kwon W, Cha SW, Yoo H, Lim S, Park JM, et al. The sonographic correlation between the sternocleidomastoid muscle thickness and the prognosis of congenital muscular torticollis. J Korean Soc Radiol 2009;60:133-8.

13. Petronic I, Brdar R, Cirovic D, Nikolic D, Lukac M, Janic D, et al. Congenital muscular torticollis in children: distribution, treatment duration and outcome. Eur J Phys Rehabil Med 2010;46:153-7.

14. Kim SJ, Park EM, Choi WK, Seo KS, Yoon JS, Lee EH. The correlation between outcome and ultrasonographic findings in congenital muscular torticollis. J Korean Acad Rehabil Med 2001;25:601-8. 